To appear in Ap. J. Letters. Subm. 2001 Mar 10; rev. 2001 Apr 16.

\title{
Low Albedos Among Extinct Comet Candidates
}

\author{
Yanga R. Fernández ${ }^{1}$, David C. Jewitt ${ }^{1}$, Scott S. Sheppard \\ Institute for Astronomy, Univ. of Hawai'i at Mānoa, \\ 2680 Woodlawn Dr., Honolulu, HI 96822 \\ yan@ifa.hawaii.edu, jewitt@ifa.hawaii.edu, sheppard@ifa.hawaii.edu
}

\begin{abstract}
We present radiometric effective radii and visual geometric albedos for six asteroids in comet-like orbits. Our sample has three of the four known retrograde asteroids (1999 $\mathrm{LE}_{31}, 2000 \mathrm{DG}_{8}$, and $\left.2000 \mathrm{HE}_{46}\right)$ and three objects ((18916) $2000 \mathrm{OG}_{44}, 2000 \mathrm{PG}_{3}$, and $2000 \mathrm{SB}_{1}$ ) on prograde but highly elliptical orbits. These measurements more than double the number of known albedos for asteroids with a Tisserand invariant in the cometary regime. We find that all six of our objects, and nine of the ten now known, have albedos that are as low as those of active cometary nuclei, which is consistent with their supposed evolutionary connection to that group. This albedo distribution is distinct from that of the whole near-Earth and unusual asteroid population, and the strong correlation between Tisserand invariant and albedo suggests there is a significant cometary contribution to this asteroid population.
\end{abstract}

Subject headings: asteroids — comets: general

\section{Introduction}

An old comet that loses all of its available volatiles or is covered by a mantle that prevents sublimation of subsurface ice will observationally appear as a near-Earth or unusual ${ }^{2}$ asteroid (NEA or UA). The dynamical lifetime of short-period comets are about 10 to 100 times longer than the devolatilization timescale (Levison \& Duncan 1994), so one expects to see such extinct comets if they do not disintegrate or collide with a planet. However there is currently no way to

\footnotetext{
${ }^{1}$ Visiting Astronomer at W. M. Keck Observatory, which is jointly operated by the California Institute of Technology and the University of California.

${ }^{2}$ The IAU Minor Planet Center currently defines "near-Earth" asteroids as those with perihelion distances below 1.3 AU, and "unusual" asteroids as those that are not near-Earth and residing neither in the Main Belt nor wholly in transjovian space.
} 
determine if a given asteroid is such a comet (short of witnessing last gasp activity), so we do not yet know the fraction of extinct comets in the asteroid population. Knowing this fraction would give clues to the physical evolution of comets and the hazard to Earth from asteroid and comet collisions.

Dynamical models of the cometary component are rendered inconclusive by the unknown extent and duration of nongravitational forces on active nuclei (Harris \& Bailey 1998). Some models (Bottke et al. 2000) can even fit the NEA population without a cometary source. Nevertheless, the existence of the now-inactive comet 107P/Wilson-Harrington, which has been observed to have a coma just once in the 51 years since discovery (Bowell 1992; Fernández et al. 1997), strongly suggests that there is a non-zero cometary contribution, and we have taken an observational tack to address the problem. A statistical indicator for the cometary origin of an asteroid comes from the albedo and orbit shape. All known albedos of cometary nuclei are low and almost all inner Solar System cometary orbits have common characteristics. Thus an asteroid in a comet-like orbit with a comet-like albedo is a good candidate extinct comet.

Nearly a dozen albedos of active cometary nuclei have been measured and all are dark, with geometric albedos ranging from 0.02 to 0.12 (Jewitt 1992; Fernández 1999). Thus we expect to see similar albedos among the extinct comet fraction of NEAs and UAs. Albedo alone is not a unique determinant of origin however since there are many non-cometary, dark asteroids in the outer Main Belt (Gradie, Chapman, \& Tedesco 1989) from which some NEAs and UAs could have come.

The Tisserand invariant $T_{J}$ (Tisserand 1896), a constant of motion in the restricted threebody problem, can be used to separate objects by dynamical class. The threshold $T_{J}=3$ separates objects coupled to $(<3)$ or decoupled from $(>3)$ Jupiter. Generally asteroids have $T_{J}>3$, Jupiterfamily (JF) comets have $2<T_{J} \leq 3$, and Halley-family (HF) and long-period (LP) comets have $T_{J} \leq 2$ (Levison 1996), though the scheme is not fail-safe. There are currently (mid-April 2001) 11 asteroids with $T_{J}<2,4$ of which are in retrograde orbits, and 131 NEAs and UAs with $2<T_{J}<3$. The total number of NEAs and UAs is currently 1,400, so $10 \%$ have $T_{J}<3$. Among NEAs alone, 82 out of 1,327 , or $6 \%$, have $T_{J}<3$. In this Letter we describe the radiometric determination of the albedos and effective radii of six $T_{J}<3$ asteroids. The Tisserand values, the contributing orbital elements, and the geometry of our observations are given in Table 1 . The new data bring to ten the total number of $T_{J}<3$ asteroids with known albedos.

\section{Observations and Reduction}

The observations span two wavelength regimes, mid-infrared (MIR) and visible (simultaneously for 4 of the 6 objects). The MIR images were obtained with the Keck I telescope using the LWS array (Jones \& Puetter 1993) in June 2000 and with the Keck II telescope using the MIRLIN array (Ressler et al. 1994) in November 2000. The visible data were obtained with the Univ. of Hawaii 2.2-m telescope using a Tek2048 CCD in July and November 2000. Table 2 gives the averages of 
the measured flux densities. All objects were point sources.

The MIR data were obtained using chopping and nodding, with throws of 4 arcsec. Nonsidereal guiding was used for each target. Flat fields were obtained by comparing staring images taken at both high and low airmass. For photometric calibration of LWS data we compared count rates to the following known $(12.5$ and $17.9 \mu \mathrm{m})$ flux densities of standard stars: $\alpha$ Lyr, 26.4 Jy and 12.9 Jy; $\sigma \mathrm{Lib}, 120.7 \mathrm{Jy}$ and $58.9 \mathrm{Jy} ; \alpha \mathrm{CrB}$, 3.64 Jy and 1.97 Jy; $\gamma$ Aql, $54.3 \mathrm{Jy}$ and $27.5 \mathrm{Jy}$. For our MIRLIN data we used the following known (11.7, 12.5, and $20.8 \mu \mathrm{m}$ ) flux densities and stars: $\gamma$ Aql, $61.7 \mathrm{Jy}, 54.3 \mathrm{Jy}$, and $20.6 \mathrm{Jy} ; \beta$ Peg, $313 \mathrm{Jy}, 277 \mathrm{Jy}$, and $107 \mathrm{Jy} ; \alpha$ Ari, $62.9 \mathrm{Jy}, 55.5$ Jy, and $21.1 \mathrm{Jy} ; \alpha \mathrm{CMi}, 59.7 \mathrm{Jy}, 52.2 \mathrm{Jy}$, and $18.8 \mathrm{Jy}$. The values are derived from the standard system of Tokunaga (1984). Comparing raw photometry over a range of airmasses let us find the extinction corrections: 0.12 and $0.35 \mathrm{mag} /$ airmass at 12.5 and $17.9 \mu \mathrm{m}$ respectively for the June data; $0.08,0.10$, and $0.40 \mathrm{mag} /$ airmass at $11.7,12.5$, and $20.8 \mu \mathrm{m}$ respectively for the November data. To maximize the signal-to-noise ratio in the photometry we used aperture corrections derived from nearby standard star radial profiles.

The visible images were obtained while guiding on a nearby star with sidereal tracking rates in July but non-sidereal rates in November. A flat field was obtained by combining images of the blank twilight sky. Flux calibration and airmass corrections were calculated by measurements of the Landolt (1992) standard stars SA 107-457, -456 and the PG 1323-086 group in July 2000, and stars SA 98-966, -1002, -L3, and -L4 in November 2000.

\section{Analysis}

The basic radiometric method to obtain an effective radius $R$ and geometric albedo $p$ is to solve two equations with these two unknowns, first done by Allen (1970) and described in detail by Lebofsky \& Spencer (1989):

$$
\begin{aligned}
F_{v i s}\left(\lambda_{v i s}\right) & =\frac{F_{\odot}\left(\lambda_{v i s}\right)}{(r / 1 \mathrm{AU})^{2}} \pi R^{2} p \frac{\Phi_{v i s}}{4 \pi \Delta^{2}}, \\
F_{m i r}\left(\lambda_{m i r}\right) & =\epsilon \int B_{\lambda}\left(T(p q, \Omega), \lambda_{m i r}\right) d \Omega R^{2} \frac{\eta \Phi_{m i r}}{4 \pi \Delta^{2}},
\end{aligned}
$$

where $F$ is the measured flux density of the object at wavelength $\lambda$ in the visible ("vis") or mid-infrared ("mir"); $F_{\odot}$ is the flux density of the Sun at Earth as a function of wavelength; $r$ and $\Delta$ are the object's heliocentric and geocentric distances, respectively; $\Phi$ is the phase function in each regime; $B_{\lambda}$ is the Planck function; $\epsilon$ is the infrared emissivity; $\eta$ is a factor to account for infrared beaming; and $T$ is the temperature, which is a function of $p$, surface planetographic position $\Omega$, and the phase integral $q$ which links the geometric and Bond albedos. For lack of 
detailed shape and rotational information - as is the case for our six objects - the modeled body is assumed to be a sphere.

The temperature is calculated using a model of the thermal behavior. Unfortunately, the thermal inertias are largely unknown so we use two widely-employed simple models that cover the extremes of thermal behavior: one for slow-rotators and one for fast. The former (a.k.a. "standard thermal model", STM) applies if the rotation is so slow (or the thermal inertia so low) that every point on the surface is in instantaneous equilibrium with the impinging solar radiation. The latter (a.k.a. "isothermal latitude model", ILM) applies if the rotation is so fast (or the thermal inertia so high) that a surface element does not appreciably cool as it spins away from local noon and out of sunlight. The extreme case occurs when the object's rotation axis is normal to the Sun-object-Earth plane.

There are other parameters to the models: $\epsilon, \eta, \Phi_{\text {mir }}, \Phi_{v i s}$, and $q$. Emissivity is close to unity and we will assume a constant value of 0.9 here. The beaming parameter is unity by definition in the ILM. In the STM, the value is known for only a few asteroids and can range from approximately 0.7 to 1.2 (Harris 1998; Harris \& Davies 1999). For our applications of the STM we assume a possible range of $0.75 \leq \eta \leq 1.25$, uniformly distributed.

For $\Phi$ we assume that the magnitude scales with the phase angle $\alpha:-2.5 \log \Phi=\beta \alpha$. In the MIR, the effect is only loosely constrained but based on earlier work with the STM (Lebofsky et al. 1986) we assume a range of $0.005 \mathrm{mag} / \mathrm{deg} \leq \beta_{\text {mir }} \leq 0.017 \mathrm{mag} / \mathrm{deg}$, uniformly distributed. For the ILM $\beta_{\text {mir }}=0$ by definition. In the visible, studied in much more detail (e.g. by Lumme \& Bowell (1981)), dark asteroids follow $\beta_{\text {vis }} \approx 0.035 \mathrm{mag} / \mathrm{deg}$, and we assume here $0.025 \mathrm{mag} / \mathrm{deg}$ $\leq \beta_{\text {vis }} \leq 0.045 \mathrm{mag} / \mathrm{deg}$. This coefficient also determines $q$, but since that has a minor effect on the modeling we will leave that parameter a constant 0.5 , the integral's value for $\beta_{v i s}=0.034$ (Allen 1973).

Our radius and albedo results from this modeling are shown in Table 3. Since we have as many or more parameters in the model than data points, useful $\chi^{2}$-distribution calculations are impossible. Instead, we found the range of valid $R$ and $p$ by sampling parameter space and declaring a good fit when each model flux density passed within 1.5- $\sigma$ of its data point. The "error bars" in Table 3 actually show the full range (not 1- $\sigma$ limits) of values that yield acceptable fits. For 1999 $\mathrm{LE}_{31}$, we used the average of the modeling results from the two nights of MIR photometry.

The STM and ILM predict very different color temperatures, providing a way to differentiate them. For $2000 \mathrm{DG}_{8}, 2000 \mathrm{OG}_{44}$, and $2000 \mathrm{SB}_{1}$, only the STM provides acceptable fits. For 2000 $\mathrm{PG}_{3}$, the ILM generally gives poorer fits and is acceptable only for a small range of radii and albedos. For $1999 \mathrm{LE}_{31}$ and $2000 \mathrm{HE}_{46}$, we only have one MIR wavelength and thus cannot find the color temperature. We note that the ILM albedos are very small, smaller than for any other Solar System object. This, combined with the generally poorer fits, implies that the objects are better interpreted as being slow rotators, although the heliocentric distance of $1999 \mathrm{LE}_{31}$ may be high enough to make it a borderline case between the two extremes (Spencer, Lebofsky, \& Sykes 
1989).

Some further caveats are notable. First, the values in Table 3 are valid in the context of the thermal models; the models represent extrema of thermal behavior and the ranges in Table 3 do not describe the systematic errors from the models themselves. However these errors are likely to be comparable to the formal errors so the values are physically meaningful. Second, rotational variation could corrupt the albedo calculations for the two asteroids with non-simultaneous MIR and visible photometry, which effectively increases the albedo errors. Third, our observations of $2000 \mathrm{PG}_{3}$ took place at $\alpha=59^{\circ}$, which is beyond the range of any measured thermal phase function, so we do not know if the standard formula for $\Phi_{m i r}$ mimics reality at such angles.

The radii of our six objects are not unusual in comparison to cometary nuclei (Meech, Hainaut, \& Marsden 2000) and other, dynamically-similar asteroids (Veeder et al. 1989), and we now turn our attention to the albedos. Figure 1 displays the albedos and Tisserand invariants of our six objects as well as all other, 42 known albedos of NEAs and UAs. Also plotted are albedos of active cometary nuclei.

Whereas previously there were only four $T_{J}<3$ asteroids on the plot, there are now ten, and all six new ones fall squarely within the cometary regime of albedos. Moreover, with these new additions we now see evidence of a trend with $T_{J}$. Ninety percent of the $T_{J}<3$ asteroids have comet-like albedos (median albedo is 0.038 ), whereas only two of the $38 T_{J}>3$ objects do (median albedo is 0.215 ). An important bias in the distribution here is that it is easier to discover shinier asteroids and thus they are overrepresented in the sample, but nevertheless the difference in distributions is quite stark.

The break in the distributions occurs near the line $T_{J}=3$, the cutoff for Jupiter coupling, which suggests a dynamical relationship with this effect. The low albedos of nearly all $T_{J}<3$ objects are consistent with a significant fraction of extinct comets among the NEA and UA population. Specifically, if $10 \%$ of NEAs and UAs have $T_{J}<3$, and $90 \%$ of those have comet-like albedos, the extinct comet candidate fraction would be approximately $9 \%$. This is a lower limit to the actual fraction of candidates because of the albedo bias mentioned above.

\section{Summary}

Using MIR and visible photometry, and employing the widely-used standard thermal model (STM) for slow rotators, we have derived new effective radii and geometric albedos for six asteroids in comet-like orbits; all six have $T_{J}<3$. We find the following:

- All six objects are dark, as dark as the albedo spread of cometary nuclei. The radii are also similar to those of active nuclei. This is consistent with a cometary origin, as if the asteroids were formerly active comets that lost all near-surface volatiles.

- For four objects we have photometry at 2 or 3 MIR wavelengths, and the STM yields an 
excellent description of the color temperature, better than the fast-rotator model.

- Plotting all 48 known NEA and UA albedos - including our 6 new ones - vs. $T_{J}$ shows a markedly sharp break, virtually a step function, at the line $T_{J}=3$.

- Eleven of the 48 objects have comet-like albedos: fully $90 \%$ (9 of 10) of the $T_{J}<3$ objects, but only $5 \%$ (2 of 38 ) of the $T_{J}>3$ objects. The median albedos $\bar{p}$ and their r.m.s. scatters are:

$$
\begin{aligned}
& \bar{p}=0.038 \pm 0.043, \text { for } T_{J} \leq 3, \text { and } \\
& \bar{p}=0.215 \pm 0.147, \text { for } T_{J}>3 .
\end{aligned}
$$

- This disparity in median albedo suggests the fraction of extinct comets among NEAs and UAs is significant (at least $9 \%$ are candidates) and that enough cometary nuclei have sufficiently long physical lifetimes to survive devolatilization without disintegrating.

It is clear that further surveys of asteroid albedos are necessary. Specifically, only 4 of the 10 $T_{J}<3$ objects are NEAs; more members of that group need to be sampled. Furthermore, with the recent explosion in asteroid discoveries and the ready availability of sensitive MIR detectors, a less biased sampling of albedos should be undertaken to obtain a confident estimate of the albedo distributions.

We are indebted to Michael Ressler for allowing MIRLIN's use on Keck and to Varoujan Gorjian for instrument support. The operation of MIRLIN is supported by an award from NASA's Office of Space Science. We thank operators Joel Aycock, Meg Whittle, Wayne Wack, and John Dvorak for their assistance. We acknowledge the JPL SSD group for their very useful "Horizons"

ephemeris program. We appreciate the help of Olivier Hainaut in improving this manuscript. This work was supported by grants to DCJ from NSF.

\section{REFERENCES}

Allen, C. W. 1973, Astrophysical Quantities (3d ed.; London: Athlone Press)

Allen, D. A. 1970, Nature, 227, 158

Bell, J. F., Hawke, B. R., \& Brown, R. H. 1988, Icarus, 73, 482

Bottke, W. F., Jedicke, R., Morbidelli, A., Petit, J.-M., \& Gladman, B. 2000, Science, 288, 2190

Bowell, E. 1992, IAU Circ. 5585

Cruikshank, D. P., \& Jones, T. J. 1977, Icarus, 31, 427

Delbo, M., Harris, A. W., Binzel, R. P., \& Davies, J. K. 2000, Bull. Amer. Astron. Soc., 32, 1000. 
Fernández, Y. R. 1999, Ph. D. Thesis, Univ. Maryland, College Park

Fernández, Y. R., McFadden, L. A., Lisse, C. M., Helin, E. F., \& Chamberlin, A. B. 1997, Icarus, 128,114

Fernández, Y. R., Meech, K. J., Lisse, C. M., A’Hearn, M. F., Pittichová, J., \& Belton, M. J. S. 2001, Icarus, submitted

Gradie, J. C., Chapman, C. R., \& Tedesco, E. F. 1989, in Asteroids II, ed. R. P. Binzel et al. (Tucson: Univ. of Arizona Press), 316

Green, S. F., Meadows, A. J., \& Davies, J. K. 1985, MNRAS, 214, 29p

Harris, A. W. 1998, Icarus, 131, 291

Harris, A. W., \& Davies, J. K. 1999, Icarus, 142, 464

Harris, N. W., \& Bailey, M. E. 1998, MNRAS, 297, 1227

Hudson, R. S., \& Ostro, S. J. 1995, Science, 270, 84

Jewitt, D. C. 1992, in Comets in the Post-Halley Era, ed. R. L. Newburn et al. (Dordrecht: Kluwer Academic), 19

Jones, B., \& Puetter, R. 1993, Proc. SPIE, 1946, 610

Landolt, A. U. 1992, AJ, 104, 340

Lebofsky, L. A., Lebofsky, M. J., \& Rieke, G. H. 1979, AJ, 84, 885

Lebofsky, L. A., \& Spencer, J. S. 1989, in Asteroids II, ed. R. P. Binzel et al. (Tucson: Univ. of Arizona Press), 128

Lebofsky, L. A., Sykes, M. V., Tedesco, E. F., Veeder, G. J., Matson, D. L., Brown, R. H., Gradie, J. C., Feierberg, M. A., \& Rudy, R. J. 1986, Icarus, 68, 239

Lebofsky, L. A., Veeder, G. J., Lebofsky, M. J., \& Matson, D. L. 1978, Icarus, 35, 336

Lebofsky, L. A., Veeder, G. J., Rieke, G. H., Lebofsky, M. J., Matson, D. L., Kowal, C., WynnWilliam, C. G., \& Becklin, E. E. 1981, Icarus, 48, 335

Levison, H. F. 1996, in ASP Conf. Ser. 173, Completing the Inventory of the Solar System, ed. T. W. Rettig \& J. M. Hahn (San Francisco: ASP), 173

Levison, H. F., \& Duncan, M. J. 1994, Icarus, 108, 18

Lumme, K., \& Bowell, E. 1981, AJ, 86, 1694 
Meech, K. J., Hainaut, O. R., \& Marsden, B. G. 2000, in Minor Bodies in the Outer Solar System, ed. A. Fitzsimmons et al. (Berlin: Springer), 75

Morrison, D., Gradie, J. C., \& Rieke G. H. 1976, Nature, 260, 691

Mottola, S., et al. 1997, AJ, 114, 1234

Pravec, P., Wolf, M., Sarounova, L., Harris, A. W., \& Davies, J. K. 1997, Icarus, 127, 441

Ressler, M. E., Werner, M. W., van Cleve, J., \& Chou, H. 1994, Exp. Astron., 3, 277

Spencer, J. R., Lebofsky, L. A., \& Sykes, M. V. 1989, Icarus, 78, 337

Tedesco, E. F. 1992, The IRAS Minor Planet Survey, Document PL-TR-92-2049, Phillips Laboratory, Hanscom Air Force Base

Tedesco, E. F., \& Gradie, J. 1987, AJ, 93, 738

Thomas, P. C., et al. 2000, Icarus, 145, 348

Tisserand, F. 1896, Traité de Mécanique Céleste, Vol. 4 (Paris, Gauthier-Villars)

Tokunaga, A. 1984, AJ, 89, 172

Veeder, G. J., Hanner, M. S., Matson, D. L., Tedesco, E. F., Lebofsky, L. A., \& Tokunaga, A. T. 1989, AJ, 97, 1211 
Table 1: Orbits and Observing Geometry

\begin{tabular}{ccccccccc}
\hline \hline Object & $a$ & $e$ & $i$ & $T_{J}$ & $\begin{array}{c}\text { UT Date } \\
(\mathrm{A} . \mathrm{D} .2000)\end{array}$ & $\begin{array}{c}r \\
(\mathrm{AU})\end{array}$ & $\begin{array}{c}\Delta \\
(\mathrm{AU})\end{array}$ & $\begin{array}{c}\alpha \\
\left(^{\circ}\right)\end{array}$ \\
\hline $1999 \mathrm{LE}_{31}$ & 8.16 & 0.472 & 152 & -1.31 & Jun 22 & 5.238 & 5.118 & 11.2 \\
$"$ & $"$ & $"$ & $"$ & $"$ & Jun 23 & 5.240 & 5.137 & 11.2 \\
$"$ & $"$ & $"$ & $"$ & $"$ & Jul 2 & 5.267 & 5.311 & 11.0 \\
$2000 \mathrm{HE}_{46}$ & 24.3 & 0.903 & 158 & -1.51 & Jun 23 & 2.526 & 2.470 & 23.5 \\
$"$ & $"$ & $"$ & $"$ & $"$ & Jul 2 & 2.562 & 2.689 & 22.2 \\
$2000 \mathrm{DG}_{8}$ & 10.8 & 0.793 & 129 & -0.62 & Nov 8 & 2.319 & 1.728 & 22.9 \\
$2000 \mathrm{OG}_{44}$ & 3.88 & 0.581 & 7.33 & 2.74 & Nov 8 & 1.665 & 0.920 & 30.6 \\
$2000 \mathrm{PG}_{3}$ & 2.83 & 0.859 & 20.5 & 2.55 & Nov 8 & 1.065 & 0.929 & 59.1 \\
$2000 \mathrm{SB}_{1}$ & 3.34 & 0.541 & 22.2 & 2.81 & Nov 8 & 1.554 & 0.673 & 25.6 \\
\hline
\end{tabular}

Note. $-a=$ semimajor axis, $e=$ eccentricity, $i=$ inclination, $r=$ heliocentric distance at time of observation, $\Delta$ $=$ geocentric distance at time of observation, and $\alpha=$ phase angle at time of observation. 
Table 2: Asteroid Photometry

\begin{tabular}{|c|c|c|c|c|c|}
\hline Object & $\begin{array}{c}\text { UT Date } \\
\text { (A.D.2000) } \\
\end{array}$ & UT Time & $\begin{array}{c}\text { Wavelength } \\
(\mu \mathrm{m})^{\mathrm{a}}\end{array}$ & $\begin{array}{c}\text { Flux Density } \\
(\mathrm{mJy}, \mathrm{Jy}, \text { or mag) }\end{array}$ & $\begin{array}{c}\text { Meas } \\
\mathrm{b}\end{array}$ \\
\hline $1999 \mathrm{LE}_{31}$ & Jun 22 & $07: 11-07: 27$ & 12.5 & $6.35 \pm 0.78 \mathrm{mJy}$ & 2 \\
\hline$"$ & Jun 23 & $06: 32-06: 49$ & 12.5 & $5.65 \pm 0.71 \mathrm{mJy}$ & 2 \\
\hline$"$ & Jun 23 & $06: 17$ & 17.9 & $\leq 57 \mathrm{mJy}^{\mathrm{c}}$ & 1 \\
\hline$"$ & Jul 2 & $07: 32$ & 0.65 & $20.44 \pm 0.05 \mathrm{mag}$ & 1 \\
\hline $2000 \mathrm{HE}_{46}$ & Jun 23 & 08:01-08:20 & 12.5 & $29.4 \pm 1.9 \mathrm{mJy}$ & 2 \\
\hline$"$ & Jul 2 & 06:16 & 0.65 & $20.11 \pm 0.02 \mathrm{mag}$ & 1 \\
\hline $2000 \mathrm{DG}_{8}$ & Nov 8 & $13: 06-13: 12$ & 11.7 & $0.382 \pm 0.013 \mathrm{Jy}$ & 4 \\
\hline$"$ & Nov 8 & $12: 35-13: 38$ & 12.5 & $0.411 \pm 0.037 \mathrm{Jy}$ & 13 \\
\hline$"$ & Nov 8 & $12: 43-13: 28$ & 20.8 & $0.71 \pm 0.15 \mathrm{Jy}$ & 3 \\
\hline$"$ & Nov 8 & $12: 12-12: 47$ & 0.65 & $16.826 \pm 0.016 \mathrm{mag}$ & 8 \\
\hline $2000 \mathrm{OG}_{44}$ & Nov 8 & 06:07-06:12 & 12.5 & $0.766 \pm 0.032 \mathrm{Jy}$ & 3 \\
\hline , & Nov 8 & $06: 14-06: 20$ & 20.8 & $0.739 \pm 0.079 \mathrm{Jy}$ & 4 \\
\hline$"$ & Nov 8 & $06: 15-06: 17$ & 0.65 & $16.39 \pm 0.01 \mathrm{mag}$ & 2 \\
\hline $2000 \mathrm{PG}_{3}$ & Nov 8 & 04:39-05:13 & 12.5 & $0.650 \pm 0.094 \mathrm{Jy}$ & 6 \\
\hline$"$ & Nov 8 & 04:43-05:15 & 20.8 & $0.60 \pm 0.12 \mathrm{Jy}$ & 2 \\
\hline$"$ & Nov 8 & $05: 11-05: 16$ & 0.65 & $17.857 \pm 0.013 \mathrm{mag}$ & 2 \\
\hline $2000 \mathrm{SB}_{1}$ & Nov 8 & 11:07-11:09 & 11.7 & $1.112 \pm 0.035 \mathrm{Jy}$ & 2 \\
\hline$"$ & Nov 8 & $11: 02-11: 48$ & 12.5 & $1.139 \pm 0.061 \mathrm{Jy}$ & 8 \\
\hline$"$ & Nov 8 & $11: 11-11: 40$ & 20.8 & $1.48 \pm 0.35 \mathrm{Jy}$ & 3 \\
\hline$"$ & Nov 8 & $11: 15-11: 18$ & 0.65 & $16.27 \pm 0.01 \mathrm{mag}$ & 2 \\
\hline
\end{tabular}

a "0.65" refers to $\mathrm{R}$ band.

$b$ "Meas." signifies the number of measurements that were used to calculate the value in the "Flux Density" column.

${ }^{c}$ This is a $3-\sigma$ upper limit. 
Table 3. Effective Radii and Geometric Albedos ${ }^{\mathrm{a}}$

\begin{tabular}{ccccc}
\hline \hline \multirow{2}{*}{ Object } & \multicolumn{2}{c}{ Slow Rotator Model } & \multicolumn{2}{c}{ Fast Rotator Model } \\
\cline { 2 - 5 } & Eff. Radius (km) & Geom. Albedo & Eff. Radius (km) & Geom. Albedo \\
\hline $1999 \mathrm{LE}_{31}$ & $9.05_{-2.71}^{+4.04}$ & $0.031_{-0.020}^{+0.030}$ & $23.37_{-2.67}^{+2.93}$ & $0.0041_{-0.0014}^{+0.0018}$ \\
$2000 \mathrm{HE}_{46}$ & $3.55_{-0.78}^{+1.10}$ & $0.023_{-0.021}^{+0.013}$ & $6.36_{-0.28}^{+0.29}$ & $0.0067_{-0.0022}^{+0.0021}$ \\
$2000 \mathrm{DG}_{8}$ & $8.64_{-1.83}^{+2.26}$ & $0.027_{-0.022}^{+0.025}$ & $\mathrm{~b}$ & $\mathrm{~b}$ \\
$2000 \mathrm{OG}_{44}$ & $3.87_{-0.40}^{+0.50}$ & $0.038_{-0.018}^{+0.017}$ & $\mathrm{~b}$ & $\mathrm{~b}$ \\
$2000 \mathrm{PG}_{3}$ & $3.08_{-0.95}^{+1.42}$ & $0.021_{-0.017}^{+0.031}$ & $3.49_{-0.19}^{+0.21 \mathrm{c}}$ & $0.015_{-0.009}^{+0.007 \mathrm{c}}$ \\
$2000 \mathrm{SB}_{1}$ & $3.57_{-0.62}^{+0.92}$ & $0.019_{-0.010}^{+0.015}$ & $\mathrm{~b}$ & $\mathrm{~b}$ \\
\hline
\end{tabular}

${ }^{a}$ With full ranges of acceptable values (not 1- $\sigma$ limits).

${ }^{b}$ The fast model gives unacceptable fits for these objects.

${ }^{\mathrm{c}}$ The fast model gives generally poorer fits and is acceptable for only a small spread of radii and albedos. 
Fig. 1.- Plot of Tisserand invariant vs. all known geometric albedos for $T_{J}<3$ NEAs and UAs (green circles), $T_{J}>3$ NEAs and UAs (blue hourglasses), and comets (red squares). The six objects presented in this Letter are marked as filled circles with estimated 1- $\sigma$ errors. A heavy vertical line marks the dynamical boundary $T_{J}=3$ (see $\S 1$ ). Of the 48 total asteroids plotted, 11 have comet-like albedos, or $23 \%$, but fully $90 \%$ (9 of 10) of those with $T_{J}<3$ have comet-like albedos. This is consistent with a cometary origin for those asteroids and a significant cometary contribution to that dynamical group of asteroids. The plotted data were obtained from this work, Morrison, Gradie, \& Rieke (1976), Cruikshank \& Jones (1977), Lebofsky et al. (1978), Lebofsky et al. (1979), Lebofsky et al. (1981), Green, Meadows, \& Davies (1985), Tedesco \& Gradie (1987), Bell, Hawke, \& Brown (1988), Veeder et al. (1989), Tedesco (1992), Hudson \& Ostro (1995), Mottola et al. (1997), Pravec et al. (1997), Harris (1998), Thomas et al. (2000), Harris \& Davies (1999), Fernández (1999), Delbo et al. (2000), and Fernández et al. (2001). 


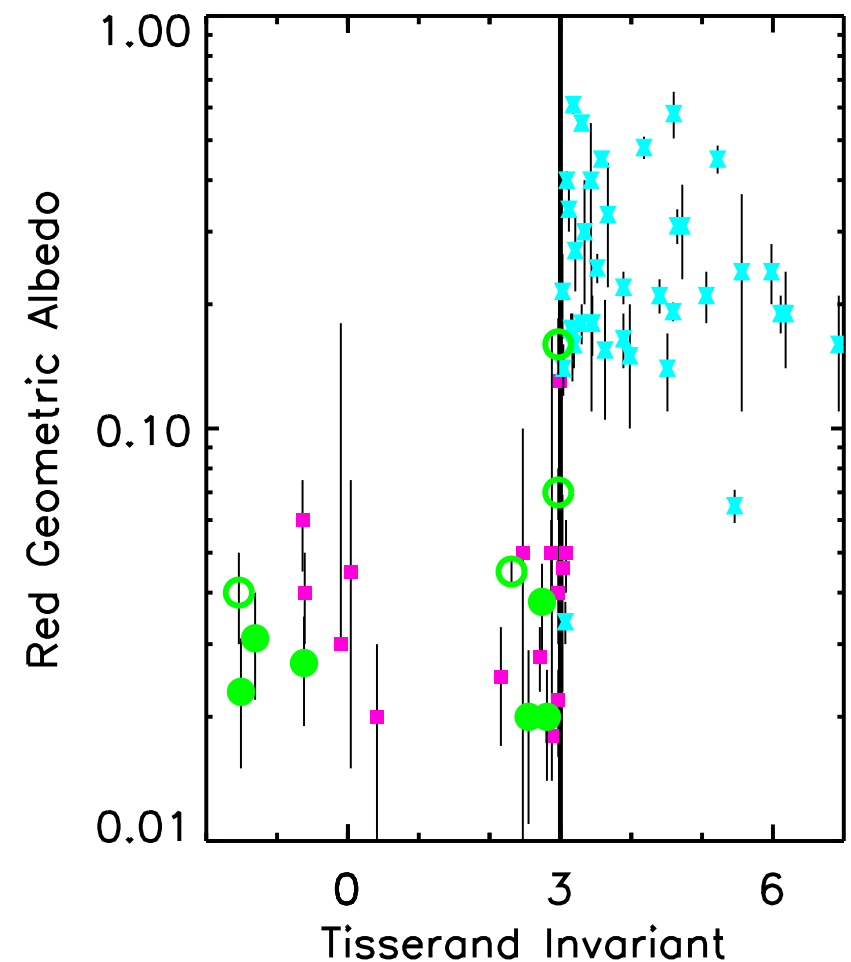

